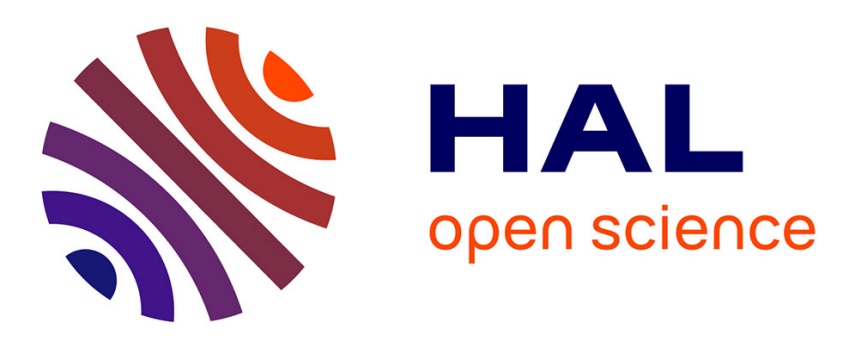

\title{
Self-excited void instability during dust particle growth in a dusty plasma
}

Lénaïc Couëdel, Maxime Mikikian, Alexander A. Samarian, Laifa Boufendi

\section{To cite this version:}

Lénaïc Couëdel, Maxime Mikikian, Alexander A. Samarian, Laifa Boufendi. Self-excited void instability during dust particle growth in a dusty plasma. Physics of Plasmas, 2010, 17, pp.083705. 10.1063/1.3479831 . hal-00502759

\section{HAL Id: hal-00502759 \\ https://hal.science/hal-00502759}

Submitted on 1 Sep 2010

HAL is a multi-disciplinary open access archive for the deposit and dissemination of scientific research documents, whether they are published or not. The documents may come from teaching and research institutions in France or abroad, or from public or private research centers.
L'archive ouverte pluridisciplinaire HAL, est destinée au dépôt et à la diffusion de documents scientifiques de niveau recherche, publiés ou non, émanant des établissements d'enseignement et de recherche français ou étrangers, des laboratoires publics ou privés. 


\title{
Self-excited void instability during dust particle growth in a dusty plasma
}

\author{
L. Couëdel ${ }^{1,2,3},{ }^{*}$ M. Mikikian ${ }^{1}$, A.A. Samarian ${ }^{2}$, and L. Boufendi ${ }^{1}$ \\ ${ }^{1}$ GREMI (Groupe de Recherches sur l'Énergétique des Milieux Ionisés), \\ CNRS/Université d'Orléans, 14 rue d'Issoudun, 45067 Orléans Cedex 2, France \\ 2 School of Physics A28, The University of Sydney, NSW 2006, Australia \\ 3 Max-Planck-Institut für extraterrestrische Physik, \\ Giessenbachstrasse, 85748 Garching, Germany.
}

(Dated: September 1, 2010)

\begin{abstract}
A new kind of void instability in a complex plasma is described. This instability is directly linked to the growth of a new generation of dust particles inside the void. It consists of slow contraction and expansion sequences of the void size which frequency and amplitude evolve while the new dust particles are growing.
\end{abstract}

PACS numbers: 52.27.Lw

\section{INTRODUCTION}

Dusty or complex plasmas are partially ionised gases composed of neutral atoms, electrons, ions and charged dust particles. Typical laboratory dust size range goes from nanometres to tens of micrometers [1]. In laboratory plasmas, the dust particles are electrically charged due to their interactions with the ions and the electrons of the plasma. Due to a higher mobility of electrons, the dust particle charge is negative. These dust particles can be either injected or grown directly in the plasma. Injected dust particles are generally bigger than the grown ones and on ground-based experiments, they are confined in the sheath region where the action of the electric field can balance the gravity [2]. On the contrary, fine dust particles can be grown directly inside the plasma (by sputtering [3] or using chemically active gas [4]) and can fill up the whole inter-electrode region.

In laboratory discharges, the dust particles generally acquire an important electric charge (typically thousands of elementary charges for micrometre size dust particles in a plasma with electron temperature $T_{e} \sim 1-2 \mathrm{eV}$ and electron density $n_{e} \sim 10^{8}-10^{9} \mathrm{~cm}^{-3}$ ). When the coupling parameter is large enough $(\Gamma \gg 1)$, self-organised structures are often observed in complex plasma and can be studied at the kinetic level thanks to the large size of the dust particles which make them easy to track. Such structures are for example plasma crystals $[5,6]$, dust waves $[7,8]$, etc. Under certain conditions, a dust free region, called a "void", can appear in the centre of the discharge. Voids are observed in ground-based experiments as well as in microgravity experiments [9-17]. They are due to a balance between the outward ion drag force onto the particles and the inward electric force [1824]. Voids can exhibit instabilities such as the heartbeat instability which consists of successive contractions and expansions of the void size $[10,11,25,26]$.

In discharges where the dust particles are grown, a cyclic

*Electronic address: 1couedel@physics.usyd.edu.au formation of dust particles inside the plasma bulk can occur if the discharge parameters are suitable [3, 27-29]. It has been reported that new generations of dust particles grow in the void region in sputtering discharges [3] or reactive plasmas [27-29]. Instabilities are often observed during the growth of the first generation [19, 30-32]. It has also been reported that instabilities can appear during the growth of successive generations in chemically active plasmas [33]. Instabilities of the void occurring during the growth of a new generation of dust particles in a sputtering discharge have also been reported [34] but never been described in details.

In this paper, we report the first detailed experimental observation of a void instability occurring during the growth of a new generation of dust particles (we propose the name of "delivery instability"). This instability consists of successive slow contractions and expansions of the void size with typical frequencies lower than the ones reported for the heartbeat instability [11] and stops when new particles are visible inside the former void. A new void then appears inside the new dust particle cloud and the instability can start again during the growth of the next generation.

\section{EXPERIMENTAL SET-UP}

The work presented here was performed in the PKENefedov (Plasma Kristall Experiment) chamber designed for microgravity experiments [35] (see Fig.1). It is a rf discharge operating in push-pull excitation mode. It consists of $4 \mathrm{~cm}$ diameter parallel electrodes separated by 3 $\mathrm{cm}$. The injected power varies in the range $0-4 \mathrm{~W}$. Dust particles were grown in an argon plasma $(0.2-2$ mbar) by sputtering of polymer layer deposited on the electrodes from previously injected dust particles $(3.4 \mu \mathrm{m}$, melamine formaldehyde). The amplitude of the discharge current was monitored during the experiments. A detailed description of this experiment and previous results are presented in Ref $[3,10,35]$.

A thin laser sheet perpendicular to the electrodes illuminated the dust particles. A fast CCD camera (Mikrotron 


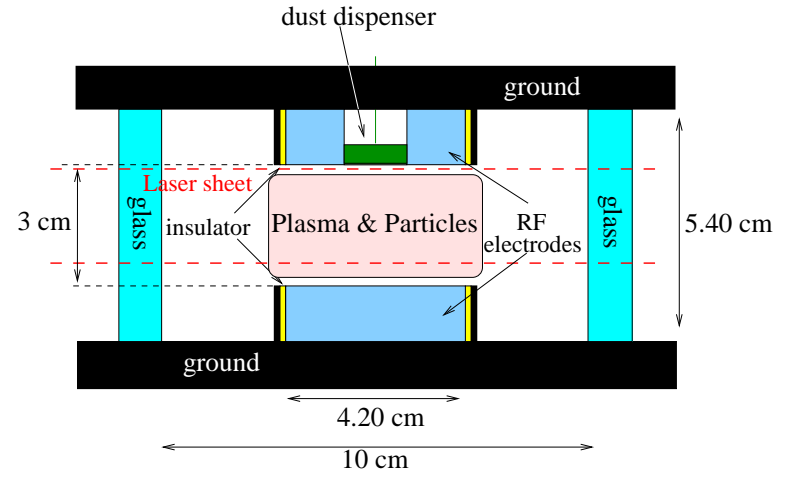

FIG. 1: (Colour online) Schematic of the PKE-Nefedov chamber.

MC 1310 or SciTech SpeedCAM MiniVis ECO-1) was used to record the plasma glow light or the scattered laser light. In order to record the laser scattered light, the camera was placed at a small angle $\left(\sim 30^{\circ}\right)$ with respect to the the laser sheet. It allowed direct observations of the dust particles at an early stage of their growth as scattering is more efficient in the forward direction. An interference filter was placed in front of the camera in order to reduce the light emitted by the plasma. In order to record the plasma glow, the camera was facing the PKE chamber without interference filter, focussed on the centre of the discharge and the laser sheet was switched off.

\section{RESULTS AND DISCUSSION}

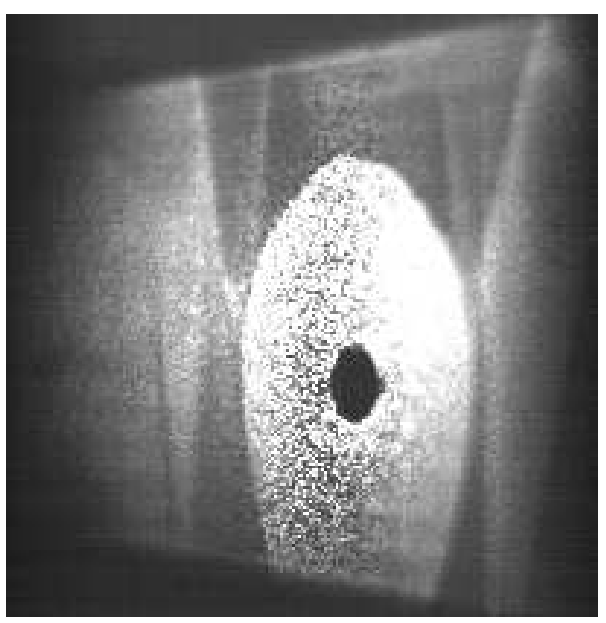

FIG. 2: Dust cloud in the PKE-Nefedov chamber.

After dust particle growth, the dust particle cloud is often in the following state (see Fig.2): a void in the centre of the chamber is surrounded by a cloud of dust particles. The cloud can be composed of different dust particle generations which are clearly separated. When the discharge parameters are suited to dust particle growth, new generations of dust particle can regularly grow in the void region. Under specific conditions (not defined yet), it can trigger an instability of the void region: the delivery instability.

The delivery instability starts with a sudden expansion of the void size followed by a contraction to approximately its original size. An expansion-contraction sequence (ECS) of the delivery instability is shown in Fig.3. It presents images extracted from high speed video imaging (500 frames per second (fps)) of the dust cloud during the delivery instability. As can be seen, the expansion in the vertical direction is more pronounced than in the horizontal direction. Fig.4 presents the time evolution of the

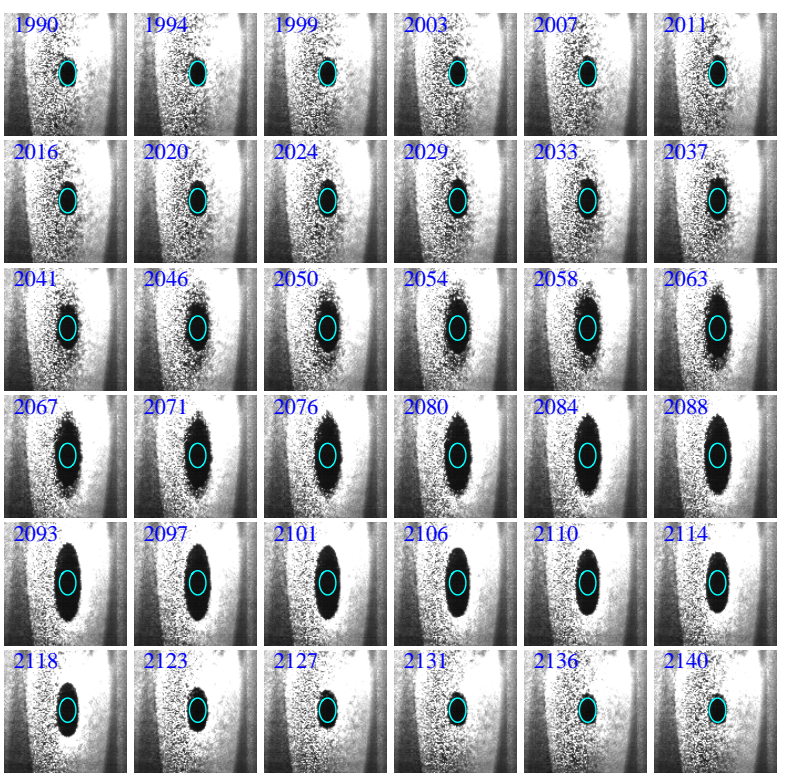

FIG. 3: (Colour online) Evolution of the void during one expansion-contraction sequence of the delivery instability. The frame are extracted from a video at $500 \mathrm{fps}$. Frame numbers are written on the upper left corner. The blue ellipse delineates the position of the stable open void.

central column going through the void extracted from the data presented in Fig.3. Time is given by the frame number on the $x$-axis ( 1 frame is $2 \mathrm{~ms}$ ) and the column profile is given in the $y$-axis. During the delivery instability, the maximum void size reached after an expansion phase increases with time. Interestingly, the minimum void size reached after a contraction also increases but to a lesser extent. Thus, it is clear that the amplitude of the void oscillation increases with time. This is one of the main differences with the fully developed heartbeat instability where the successive contraction-expansion sequences (CES) are roughly similar. It is also worth noting that the expansion time is longer than the contraction time. This is also the case during the heartbeat instability in which the characteristic times are however much shorter than those of the delivery instability (see Ref.[11]). For example, a full ECS of the delivery instability lasts for 


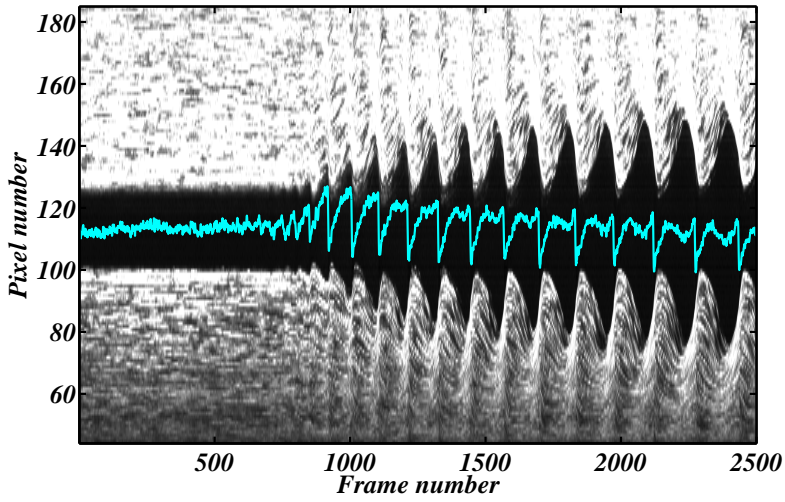

(a)

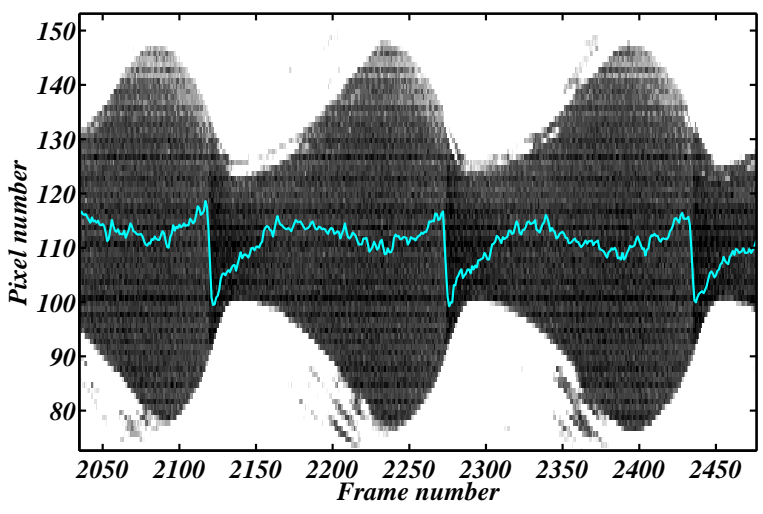

(b)

FIG. 4: (Colour online) a) Evolution of the central column going through the void (frame rate: $500 \mathrm{fps}$ ). b) Zoom of a) with enhanced contrast. The plasma glow luminosity was not totally stopped by the interference filter. It allowed us to visualise major glow luminosity fluctuation inside the void (sum of pixel intensities in the stable void region - blue lines) at the same time as the dust cloud.

hundreds of milliseconds while a full CES of the heartbeat instability lasts for at most a few tens of milliseconds. In Fig.4, the evolution of the residual plasma glow light in the void region passing through the interference filter is also shown. During the instability, the emitted light follows a complicated pattern: in early oscillations the maximum light intensity is well above the mean value recorded for the stable void while for the latter oscillations, this maximum value is comparable. Moreover, the glow variation in early oscillations are larger than in the latter one. It can also be seen that that the void contraction starts before the central glow faints.

As can be seen in Fig.5, both frequency and amplitude of the instability evolve with time. These changes must be linked to the evolution of the plasma and dust parameters during the instability. During the first oscillations, amplitude and frequency vary strongly as does the plasma glow inside the void. According to OML theory, the charge on dust particles is proportional to their radius [1]. As the new particles are getting larger, their electric charge is increasing and hence the repulsion force

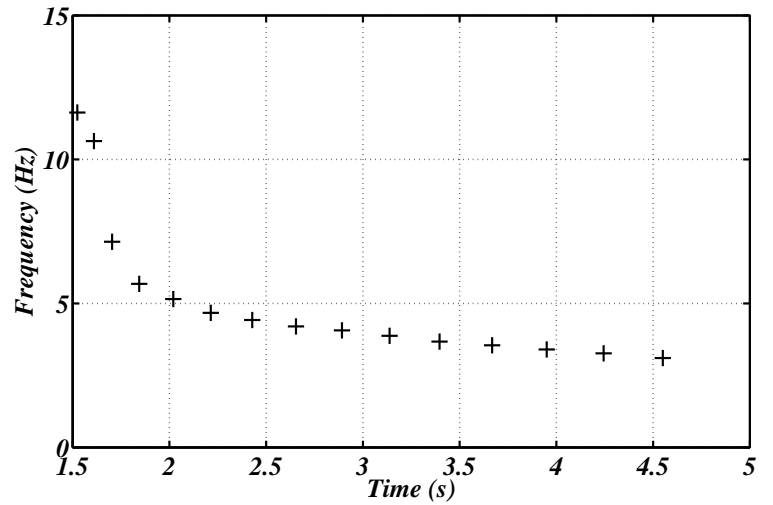

(a)

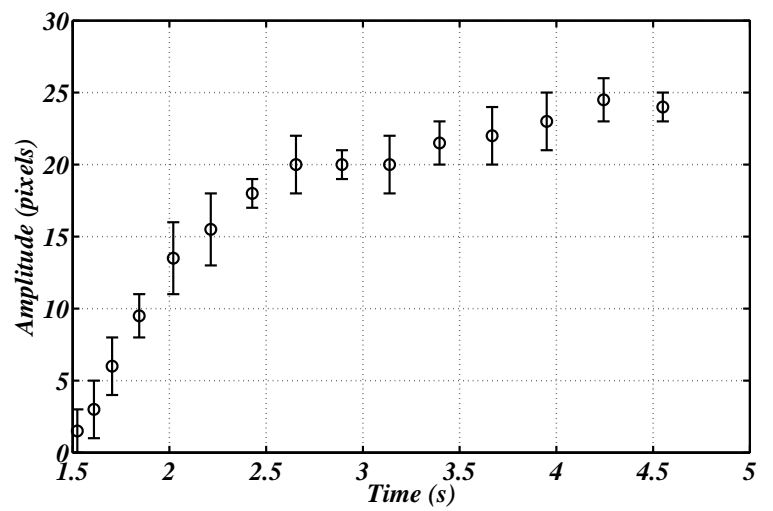

(b)

FIG. 5: (a) Evolution of the delivery instability frequency. (b) Evolution of the delivery instability amplitude (1 pixel $100 \mu \mathrm{m})$. The data were extracted from the same experiment as in Figs. 3 and 4.

between the dust particles in the new cloud is getting stronger as well as the repulsion with the old particle cloud. Consequently, the growing particles occupy more volume in the plasma and it might explain why the amplitude of the oscillation is increasing. It was reported in a sputtering discharge that dust particle density decreases at a rate $\sim \frac{1}{50} \mathrm{~s}^{-1}$ at most [19] and, the time averaged dust particle density can therefore be considered in first approximation more or less constant for the duration of the delivery instability (the density of growing dust particles was also reported to be more or less constant after few seconds in silane discharges [36]). As the dust plasma frequency at constant dust density is inversely proportional to the square root of the dust particle mass and proportional to its charge, the frequencies associated with dust motion in the growing cloud tend to decrease and might be linked to the observed drop of the void oscillation frequency. The delivery instability ends when the new generation of dust particles reaches critical dimensions. Soon after the void stops oscillating (typically 5 to 10 seconds), the new cloud becomes visible to the naked eye. A new delivery instability can eventually be triggered again when a new generation of 


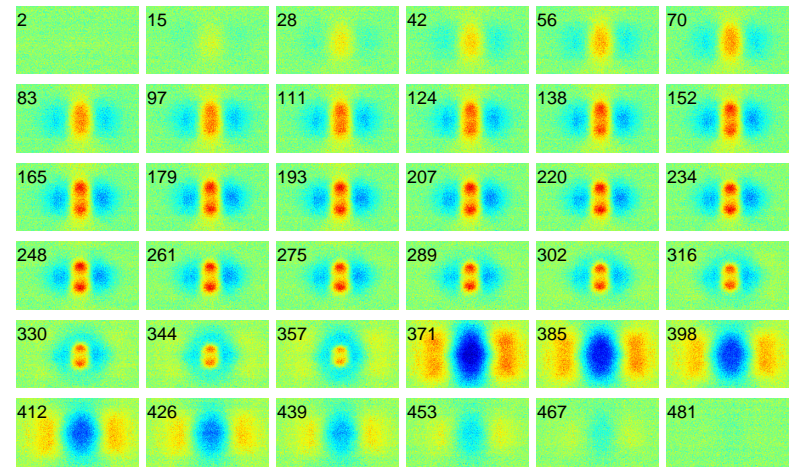

FIG. 6: (Colour online) Evolution of the glow during one expansion-contraction sequence of a delivery instability (in false colour from blue to red). In order to improve the visibility of the luminosity variations, frame 1 was taken as the reference image and has been subtracted from every other frames. Frame numbers are written on the upper left corner (frame rate: $1789 \mathrm{fps}$ )

dust starts to grow.

In order to see the global evolution of the plasma glow, the emitted light was recorded over the whole interelectrode space. In Fig.6, frames extracted from a video (1,789 fps) during a delivery instability are presented. It shows the evolution of the plasma glow luminosity during one expansion-contraction sequence of the delivery instability (not during the same experiment as in Figs. 3-5). The plasma glow starts to increase in the centre before it splits in two vertically aligned luminous glow regions which then converge towards each other before they suddenly disappear and the outer part of the plasma becomes brighter. This can be directly correlated to Fig. 4(b): the plasma light (integrated over the region corresponding to the stable void) first increase corresponding to the initial central glow increase; then it decreases due to the splitting of the glow on the upper and lower part of the oscillating void; finally, it increases again due to the convergence of the enhanced glow regions during the contraction of the void. When the situation reverses to dark centre and brighter edges, the void continues to shrink until it reaches its minimum dimensions. The plasma glow distribution returns then to its original configuration and a new expansion-contraction starts again. These effects are clearly seen in Fig.7. The discharge current (Fig.7) during the instability also exhibits interesting features: the first increase corresponds to an increase of luminosity in the plasma centre. Then the current decreases slowly while the two regions of enhanced luminosity are formed and converge toward each other. The abrupt decrease corresponds to a fast luminosity decrease in the centre while the plasma edges become brighter. The current decrease indicates that the total ionisation in the plasma decreases although localised regions of high ionisation (luminosity) exist.

The fact that the enhanced central glow luminosity
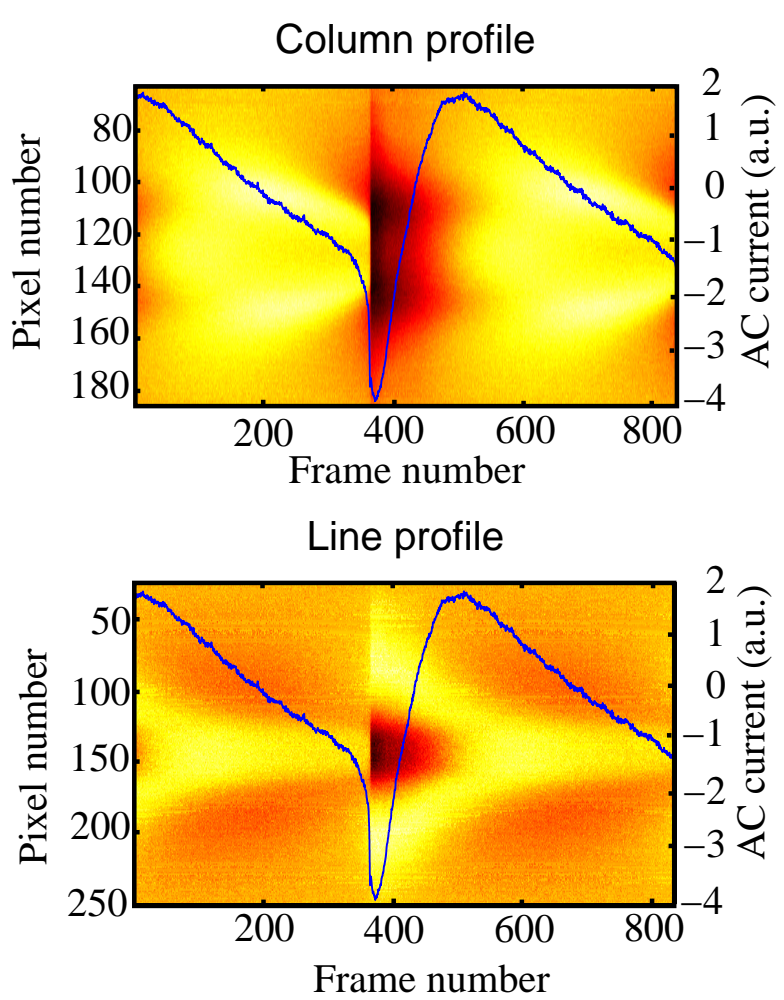

FIG. 7: (Colour online) Evolution of glow luminosity extracted from the data presented in Fig.6 (in false colour from dark red to bright yellow). Top: Evolution of the central column luminosity. Bottom: Evolution of the central line luminosity (frame rate: $1789 \mathrm{fps}$ ). In both cases, the time average value of each pixels has been subtracted in order to enhance luminosity variations. The blue line superimposed on each picture represents the discharge current (AC component, arbitrary units) during the delivery instability. Typically variations of the current during one oscillation are around $1 \%$ of its mean DC value.

(generally associated to a higher ionisation rate in the void region $[\mathbf{1 8}, \mathbf{1 9}, \mathbf{3 7}]$ ) splits into two distinctive regions during the delivery instability (Fig.6) tends to indicate that a cloud of very small dust particles in the centre of the void prevents a high ionisation rate due to the loss of charge carriers on the dust particle. The complex distribution of the enhanced ionisation areas as well as the presence of the inner dust particle cloud must result in complex ion drag and electrostatic force directions and magnitudes. However when two distinctive regions of enhanced ionisation exist in the upper and lower parts of the void, the confining forces become dominant as the void starts to contract (Fig.4(b)). It is nevertheless difficult to conclude which of the ion drag force or the electrostatic force is the dominant force due to the complexities exhibited by the system. Moreover, it should be noted that the growing particles might also affect the electron temperature inside the void and thus the glow luminosity due to excitation of the neutral atoms (it was shown in reactive plasma 
that the electron temperature increases during the growth of dust particles [38]), increasing further the complexity of the system.

\section{CONCLUSION}

In conclusion, a new kind of void instability in complex plasmas has been described. The delivery instability is directly linked to the growth of a new generation of dust particles inside the void which trigger strong oscillations of its size. We showed that the void dynamics as well as the glow evolution follows a very different pattern than the one exhibited during the well-known heartbeat instability [11]. However, the nature of the coupling between the growing dust cloud and the surrounding plasma and dust particles is still under investigation as many features of the instability such as the spatio-temporal evolution of the ionisation processes are not fully understood.

\section{Acknowledgments}

The authors would like to thank S. Dozias and B. Dumax for electronic support, J. Mathias for optical support and Y. Tessier for experimental support. The PKE-Nefedov chamber has been made available by the Max-Planck-Institute for Extraterrestrial Physics, Germany, under the funding of DLR/BMBF under grants No.50WM9852. This work was partially supported by CNES under contract 793/2000/CNES/8344 and by the Australian Research Council and the University of Sydney under grant DP0773967. This work was also partially supported by the French-Australian integrated research program (FAST) of the French foreign affairs ministry and the International Science Linkages established under the Australian Government's innovation statement, Backing Australia's Ability'.
[1] A. Bouchoule, Dusty Plasmas: Physics, Chemistry and Technological impacts in Plasma Processing (Wiley, New York, 1999).

[2] A. A. Samarian and B. W. James, Phys. Lett. A 287, 125 (2001).

[3] M. Mikikian, L. Boufendi, A. Bouchoule, H. M. Thomas, G. E. Morfill, A. P. Nefedov, V. E. Fortov, and the PKENefedov team, New J. Phys. 5, 19 (2003).

[4] M. Cavarroc, M. Mikikian, G. Perrier, and L. Boufendi, Appl. Phys. Lett. 89, 013107 (2006).

[5] H. M. Thomas and G. E. Morfill, Nature 379, 806 (1996).

[6] L. Couëdel, V. Nosenko, S. K. Zhdanov, A. V. Ivlev, H. M. Thomas, and G. E. Morfill, Phys. Rev. Lett. 103, 215001 (2009).

[7] A. Barkan, R. L. Merlino, and N. D'Angelo, Phys. Plasmas 2, 3563 (1995).

[8] S. Nunomura, S. Zhdanov, D. Samsonov, and G. Morfill, Phys. Rev. Lett. 94, 045001 (2005).

[9] M. Kretschmer, S. A. Khrapak, S. K. Zhdanov, H. M. Thomas, G. E. Morfill, V. E. Fortov, A. M. Lipaev, V. I. Molotkov, A. I. Ivanov, and M. V. Turin, Phys. Rev. E 71, 056401 (2005)

[10] M. Mikikian and L. Boufendi, Phys. Plasmas 11, 3733 (2004).

[11] M. Mikikian, L. Couëdel, M. Cavarroc, Y. Tessier, and L. Boufendi, New J. Phys. 9, 268 (2007).

[12] G. E. Morfill, H. M. Thomas, U. Konopka, H. Rothermel, M. Zuzic, A. Ivlev, and J. Goree, Phys. Rev. Lett. 83, 1598 (1999).

[13] G. Praburam and J. Goree, Phys. Plasmas 3, 1212 (1996).

[14] A. M. Lipaev, S. A. Khrapak, V. I. Molotkov, G. E. Morfill, V. E. Fortov, A. V. Ivlev, H. M. Thomas, A. G. Khrapak, V. N. Naumkin, A. I. Ivanov, S. E. Tretschev, G. I. Padalka, Phys. Rev. Lett. 98, 265006 (2007).

[15] E. Thomas, B. M. Annaratone, G. E. Morfill, and H. Rothermel, Phys. Rev. E 66, 016405 (2002).

[16] H. M. Thomas, G. E. Morfill, V. E. Fortov, A. V. Ivlev, V. I. Molotkov, A. M. Lipaev, T. Hagl, H. Rothermel,
S. A. Khrapak, R. K. Suetterlin, M. Rubin-Zuzic, O. F. Petrov, V. I. Tokarev, S. K. Krikalev, New J. Phys. 10, 033036 (2008).

[17] S. K. Zhdanov, M. Schwabe, R. Heidemann, R. Sütterlin, H. M. Thomas, M. Rubin-Zuzic, H. Rothermel, T. Hagl, A. V. Ivlev, G. E. Morfill, V. I. Molotkov, A. M. Lipaev, O. F. Petrov, V. E. Fortov, T. Reiter, New J. Phys. 12, 043006 (2010).

[18] J. Goree, G. E. Morfill, V. N. Tsytovich, and S. V. Vladimirov, Phys. Rev. E 59, 7055 (1999).

[19] D. Samsonov and J. Goree, Phys. Rev. E 59, 1047 (1999).

[20] K. Avinash, A. Bhattacharjee, and S. Hu, Phys. Rev. Lett. 90, 75001 (2003).

[21] V. N. Tsytovich, S. V. Vladimirov, and G. E. Morfill, JETP 102, 334 (2006).

[22] V. N. Tsytovich, S. V. Vladimirov, and G. E. Morfill, Phys. Rev. E 70, 066408 (2004).

[23] V. N. Tsytovich, S. V. Vladimirov, G. E. Morfill, and J. Goree, Phys. Rev. E 63, 056609 (1999).

[24] S. V. Vladimirov, V. N. Tsytovich, and G. E. Morfill, Phys. Plasmas 12, 052117 (2005).

[25] M. Mikikian, M. Cavarroc, L. Couëdel, Y. Tessier, and L. Boufendi, Phys. Rev. Lett. 100, 225005 (2008).

[26] M. Mikikian, L. Couëdel, M. Cavarroc, Y. Tessier, and L. Boufendi, Threshold Phenomena in a Throbbing Complex Plasma, Phys. Rev. Lett., accepted (2010).

[27] L. Boufendi and A. Bouchoule, Plasma Sources Sci. Technol. 3, 262 (1994).

[28] M. Cavarroc, M. Mikikian, Y. Tessier, and L. Boufendi, Phys. Rev. Lett. 100, 045001 (2008).

[29] H. T. Do, G. Thieme, M. Fröhlich, H. Kersten, and R. Hippler, Contrib. Plasma Phys. 45, 378 (2005).

[30] M. Cavarroc, M. C. Jouanny, K. Radouane, M. Mikikian, and L. Boufendi, J. Appl. Phys. 99, 064301 (2006).

[31] M. Mikikian, M. Cavarroc, L. Couëdel, and L. Boufendi, Phys. Plasmas 13, 092103 (2006).

[32] D. Samsonov and J. Goree, J. Vac. Sci. Technol. A 17, 2835 (1999).

[33] M. Cavarroc, M. Mikikian, Y. Tessier, and L. Boufendi, 
Phys. Plasmas 15, 103704 (2008).

[34] M. Mikikian, L. Boufendi, and A. Bouchoule, in $30^{\text {th }}$ EPS Conf. on Contr. Fusion and Plasma Phys., edited by R. Koch and S. Lebedev (2003), vol. 27A, pp. 0-3.1B.

[35] A. P. Nefedov, G. E. Morfill, V. E. Fortov, H. M. Thomas, H. Rothermel, T. Hagl, A. V. Ivlev, M. Zuzic, B. A. Klumov, A. M. Lipaev, et al., New J. Phys. 5, 33 (2003).

[36] L. Boufendi, J. Hermann, A. Bouchoule, B. Dubreuil,
E. Stoffels, W. W. Stoffels, and M. L. deGiorgi, J. Appl. Phys. 76, 148 (1994).

[37] D. Samsonov and J. Goree, IEEE Trans. Plasma Sci. 27, 76 (1999); V. Land and W.J. Goedheer, New J. Phys. 9, 246 (2007)

[38] A. Bouchoule and L. Boufendi , Plasma Sources Sci. Technol. 3, 292 (1994). 